\title{
CYCLIC SURGERY ON KNOTS
}

\author{
SHICHENG WANG
}

(Communicated by Frederick R. Cohen)

\begin{abstract}
We get a necessary condition under which a nonsimple knot (i.e. a satellite knot) admits a nontrivial surgery producing a lens space. Interesting corollaries are: (1) if a lens space can be obtained from a nontrivial surgery on a nonsimple knot, then the order of its fundamental group is not smaller than 23; (2) any nonsimple knot admits at most one nontrivial surgery which produces a lens space.
\end{abstract}

Some topologists are interested in the problem of doing surgery on a knot complement to get a lens space. This note is devoted to providing more information on this problem. Our main result is the following:

Theorem 1. If a lens space can be obtained from a nontrivial surgery on a nonsimple knot (i.e. a satellite knot) $J(K)$, then $K$ is a torus knot and $J$ is a closed braid in a tubular neighborhood of $K$.

There are two interesting corollaries of Theorem 1.

D. Gabai proved that one cannot get a lens space with fundamental group of order 1 (i.e. $S^{3}$ ) from a nontrivial surgery on a nonsimple knot (in fact nonsimple knots have property $P$ ). J. Bailey and D. Rolfsen [BR] (also R. Fintushel and R. Stern [FS] and C. Gordon [G]) proved that some lens space with fundamental group of order 23 can be obtained from a nontrivial surgery on a nonsimple knot. The first corollary says that 23 is the smallest possible.

Corollary 1. If a lens space can be obtained from a nontrivial surgery on a nonsimple knot (i.e. a satellite knot), then the order of its fundamental group is not smaller than 23.

M. Culler, C. M. Gordon, J. Luecke, and P. Shalen [CGLS] proved that there are at most two nontrivial surgeries on a nontorus knot which produce lens spaces. Corollary 2 below says that a nontorus knot which admits two nontrivial surgeries producing lens spaces must be a hyperbolic knot. (Berge found infinitely many such nontorus knots.)

Received by the editors February 4, 1988, and in revised form, August 17, 1988.

1980 Mathematics Subject Classification (1985 Revision). Primary 57N10. 
Corollary 2. Any nonsimple knot admits at most one nontrivial surgery which produces a lens space.

Let $K$ be a nontrivial knot in $S^{3}, N(*)$ denote tubular neighborhood. $X=$ $E(K)=S^{3}-$ int $N(K)$.

Let $f: S^{1} \times D^{2} \longrightarrow N(K)$ be a homeomorphism which determines the 0frame of $K$, i.e. $f\left(S^{1} \times *\right)=0 \in H_{1}(X)$. Now choose an arbitrary orientation of $K$. Let $\lambda=f\left(S^{1} \times *\right)$ be the longitude and $\mu=f\left(* \times \partial D^{2}\right)$ be the meridian. If $r=\frac{p}{n} \in Q \cup \infty, p, n \in Z$, and $(p, n)=1$, then $(E(K), r)=(K, r)$, and the result of Dehn surgery of type $r$ on $K$ is $X \cup_{h} S^{1} \times D^{2}$, where $h: S^{1} \times \partial D^{2} \longrightarrow$ $\partial X$ is a homeomorphism such that $h\left(* \times \partial D^{2}\right)=p \mu+n \lambda \in H_{1}(\partial X)$.

$S$ denotes a solid torus. $J$ will always denote a circle in $S$ which does not lie in a ball in $S$ and is not isotopic to the center line of $S$. The winding number $w$ of $J$ is the integer $w \geq 0$ such that $H_{1}(J) \longrightarrow H_{1}(S)$ has image $w Z$. The exterior of $J$ is $Y=S-\operatorname{int} N(J)$.

Let $K$ be a knot and $f: S \longrightarrow N(K)$ as above. Then $J(K)$ denotes the knot $f(J)$ in $S^{3}$. We call $J(K)$ a nonsimple knot or a satellite knot.

Let $\alpha, \beta \in H_{1}\left(S^{1} \times \partial D^{2}\right)$ be given by $\alpha=S^{1} \times *, \beta=* \times \partial D^{2}$. Note that $H_{1}(Y)=Z \alpha \oplus Z \mu_{J}$, where $\mu_{J}$ is a meridian of $N(J)$. There exists a homeomorphism $g: S^{1} \times D^{2} \longrightarrow N(J)$ such that $g\left(S^{1} \times *\right)=w \alpha$ in $H_{1}(Y)$. Then $\lambda_{J}=g\left(S^{1} \times *\right) \in H_{1}(\partial N(J))$ is a longitude of $J$. For $r=\frac{p}{n}$, we define $(J, r)$ to be $Y \cup_{h} S^{1} \times D^{2}$, where $h: S^{1} \times \partial D^{2} \longrightarrow \partial N(J)$ is a homeomorphism such that $h\left(* \times \partial D^{2}\right)=p \mu_{J}+n \lambda_{J} \in H_{1}(\partial N(J))$. Note that for every $K$, if $f, g$ are as above, then $f g$ determines the 0 -frame of $J(K)$. Hence $(J(K), r)=X \cup(J, r)$, identified via the restriction of $f$ to $\partial(J, r)=\partial S \longrightarrow$ $\partial N(K)=\partial X$.

$Z_{n}=\frac{Z}{n Z} \cdot H_{1}(*)=H_{1}(*, Z) . \quad L(p)$ is some lens space $L(p, n) . \quad T$ is the torus. $T_{p, q}$ is the $(p, q)$ torus knot.

The proof of Theorem 1 will employ the following results:

[CGLS]: If $K$ is not a torus knot then $\pi_{1}(K, r)$ can be cyclic only if $r$ is integer. Moreover, there are at most two such integers $r$, and if there are two then they must be successive. Finally, if $\pi_{1}(K, r)=0$, then $r=1$ or -1 .

[Ga2] (see also [S]): Let $M$ be an irreducible cobordism between tori $T_{0}$ and $T_{1}$ without nonboundary parallel essential torus, $F$ be a Thurston norm minimizing surface representing an element in $H_{2}\left(M, T_{1}\right)$ which does not hit $T_{0}$. Then for all but at most one slope $r$ on $T_{0},(M, r)$ is irreducible and $F$ is still Thurston norm minimizing in $(M, r)$, where $(M, r)$ is constructed by attaching a solid torus $S$ to $T_{0}$ such that $r$ bounds a disk in $S$.

[Ga1]: Let $J$ be a knot in $S^{1} \times D^{2}$ whose geometric intersection with $* \times D^{2}$ is nonzero. If $M$ is a manifold obtained by a nontrivial surgery on $J$ and $M$ is a solid torus, then $J$ is a braid in $S^{1} \times D^{2}$. Satellite knots have property 
$P$, i.e., nontrivial surgery on a satellite knot yields a nonsimply connected manifold.

[G]: (1) $H_{1}(J, r)=Z \oplus Z_{(w, p)}$.

(2) If $(J(K), r)$ is a lens space $L(p)$ for some integer $p$, then $(J, r) \cong$ $S \# L(q)$, where $q$ is 1 or $p$, and $(J(K) ; r)=\left(K ; \frac{r}{w^{2}}\right) \# L(p)$.

Proof of Theorem 1. For a nonsimple knot $\bar{K}=J(K)$, Suppose $(J(K) ; r)$ is a lens space. By [CGLS], $r$ is an integer $p$. By [G], we have

$$
L(p)=(J(K), p)=\left(K ; \frac{p}{w^{2}}\right) \# L(q)
$$

and

$$
(J ; p)=S \# L(q) \text {. }
$$

If $q=1$, then $(J ; p)=S$. Since $J$ does not lie in a ball in $N(K)$, its geometric intersection number with a meridian disk of $N(K)$ is nonzero. As the surgery is nontrivial, by [Ga1], $J$ is a braid in $N(K)$; therefore $w \geq 2$ (otherwise $J$ is a core of $N(K)$ ). Next we claim that $K$ is a torus knot, because $w \geq 2$, $\frac{p}{w^{2}} \neq p$. If $\frac{p}{w^{2}}$ is an integer, then

$$
\left(K ; \frac{p}{w^{2}}\right) \neq L(p)
$$

since $H_{1}\left(K, \frac{p}{w^{2}}\right)=Z \frac{p}{w^{2}} \neq Z_{p}$. So $\frac{p}{w^{2}}$ is not an integer. By [CGLS], $K$ must be a torus knot. So in the case $q=1$, we are done.

If $q=p$, then $\left(K ; \frac{p}{w^{2}}\right)=S^{3}$.

Base on [Ga2], applying the same argument of the proof of Corollary 5.2 of [S] to the situation $(J, p)=S \# L(p)$, one can show that $w$ is not zero.

Furthermore, $K$ is not a torus knot (torus knots have property $P$ ) which implies that $p=w^{2}$ or $-w^{2}$ by [CGLS]. Now we have $H_{1}\left(J, \pm w^{2}\right)=Z \oplus$ $Z_{\left(w, w^{2}\right)}=Z \oplus Z_{w}$ and $\left(J, \pm w^{2}\right)=S \# L\left(w^{2}\right)$ by [G]. This implies that $w$ must be \pm 1 . But then $p= \pm 1$, so $(\bar{K}, r)=(\bar{K}, \pm 1)=S^{3}$, which contradicts the fact that satellite knots have Property $P$.

Theorem 1 is proved.

The proof of two corollaries also need the following result of L. Moser (see $[\mathrm{M}])$.

$$
\begin{aligned}
& \left(T_{s ; t} ; \frac{m}{n}\right)=L(s) \# L(t) \quad \text { if } \frac{m}{n}=s t . \\
& \left(T_{s ; t} ; \frac{m}{n}\right)=L(m) \quad \text { if } m=n s t \pm 1 .
\end{aligned}
$$

Otherwise $\left(T_{s: t} ; \frac{m}{n}\right)$ is a Seifert manifold with orbifold $S^{2}$ on which there are three branch points of order $s,|t|$, and $|n s t-m|$.

Proof of Corollary 1. Let $\bar{K}$ be a satellite knot and $(\bar{K} ; r)=L(p)$. 
By [CGLS], $r=p$. By Theorem $1, \bar{K}=J\left(T_{s, t}\right)$, where $J$ is a braid in a tubular neighborhood of the torus knot $T_{s ; t}$ with winding number $w \geq 2$. So now we have

$$
L(p)=\left(J\left(T_{s ; t}\right) ; p\right)=\left(T_{s ; t}, \frac{p}{w^{2}}\right) \# L(q) .
$$

Torus knots have property $P$, hence

$$
\left(T_{s ; t} ; \frac{p}{w^{2}}\right) \neq S^{3} .
$$

Therefore $q=1$, and we have

$$
L(p)=\left(T_{s ; t} ; \frac{p}{w^{2}}\right) .
$$

$p$ and $w$ must be coprime. By $[\mathrm{M}], p=w^{2} s t \pm 1$. The minimum st such that $T_{s ; t}$ is nontrivial is $2 \times 3$. So the minimum $p=w^{2} s t \pm 1$ is $2^{2} \times 2 \times 3-1=23$.

Proof of Corollary 2. Let $K$ be a knot such tiat $(K ; n)$ and $(K ; p)$ are lens spaces. If $K$ is a satellite knot, from the proof of Theorem 1, we see that $n, p$ are integers which satisfy $n=w^{2} s t \pm 1$, and $p=w^{2} s t \pm 1$. (Note that $w, s$, $t$, are determined by $K$ ). Obviously, for any choice of $w, s, t ; p \neq n \pm 1$. By [CGLS], this is impossible. So $K$ is a simple knot.

\section{ACKNOWLEDGMENTS}

I would like to express my gratitude to professor R. D. Edwards, Q. Zhou, and $\mathrm{Y}$. Wu for their help. I also wish to thank the referee for his comments and suggestions.

\section{REFERENCES}

[BR] J. Bailey and D. Rolfsen, An unexpected surgery construction of lens space, Pacific J. Math. 71 (1977) 295-298.

[CGLS] M. Culler, C. M. Gordon, J. Luecke, and P. B. Shalen, Dehn surgery on knots, Ann. of Math. (2) 125 (1987), 237-300.

[FS] R. Fintushel and R. Stern, Constructing lens spaces by surgery on knots, Math. Z. 175 (1980), 33-51.

[Ga1] D. Gabai, Foliations and surgery on knots, Bull. Amer. Math. Soc. 15 (1986), 83-86.

[Ga2] __ Foliations and the topology of 3-manifolds II, J. Differential Geom. 26 (1987) 461-478.

[G] C. M. Gordon, Dehn surgery and satellite knots, Trans. Amer. Math. Soc. 275 (1983), 687-708.

[M] L. Moser, Elementary surgery along a torus knot, Pacific J. Math. 38 (1971) 734-745.

[S] M. Scharlemann, Sutured manifolds and generalized Thurston norms, J. Differential Geom. (to appear).

Mathematics Department, University of California, Berkeley, Berkeley, California, 94720 